\title{
Quality Metric for Parasternal Long Axis B-Mode Echocardiograms
}

\author{
Sri-Kaushik Pavani ${ }^{1}$, Navneeth Subramanian ${ }^{1}$, Mithun Das Gupta ${ }^{1}$, \\ Pavan Annangi ${ }^{1}$, Satish C. Govind ${ }^{2}$, and Brian Young ${ }^{3}$ \\ 1 John F. Welch Technology Center, GE Global Research, Bangalore, India \\ 2 Bhagwan Mahavir Jain Heart Centre, Bangalore, India \\ ${ }^{3}$ GE Healthcare-PCS, Milwaukee, WI, USA
}

\begin{abstract}
This paper presents a method for automatically estimating the quality of Parasternal Long AXis (PLAX) B-mode echocardiograms. The purpose of the algorithm is to provide live feedback to the user on the quality of the acquired image. The proposed approach uses Generalized Hough Transform to compare the structures derived from the incoming image to a representative atlas, thereby providing a quality metric (PQM). On 133 PLAX images from 35 patients, we show: 1) PQM has high correlation with manual ratings from an expert echocardiographer 2) PQM has high correlation with contrast-to-noise ratio, a traditional indicator of image quality 3) on images with high PQM, error in automatic septal wall thickness measurement is low, and vice versa.
\end{abstract}

\section{Introduction}

Standard views in transthoracic echo are well established. But, quality of images and correct tomographic planes for accurate clinical interpretation and measurements are dependent on operator skills. Algorithms that can automatically detect quality of ultrasound images have tremendous potential to 1) standardize imaging 2) reduce scan time for users by providing real-time feedback, and 3) provide automatic mechanism to reject data of poor quality.

Assessment of image quality prior to complex post-processing is common in the field of human identification using biometric data [1. Our contribution extends this philosophy to medical ultrasound images. Note that our work is different from image quality testing of imaging systems [2] in that we propose an approach to compute image quality during live imaging rather than on phantoms.

We propose an algorithm to automatically determine the quality of Parasternal Long AXis (PLAX) B-mode echocardiograms. At the right scan plane, and with optimal instrument settings, the long axis of the left ventricle is oriented horizontally in a standard PLAX view (See Fig. 1(a)). The posterior wall, the pericardium and the septum are approximately parallel to each other. Any deviation from this is a result of an incorrect acquisition or sub-optimal instrument settings. For example, the poor quality image shown in Fig. 1(b) could be due to sub-optimal instrument settings such as gain, dynamic range, or time-gain 

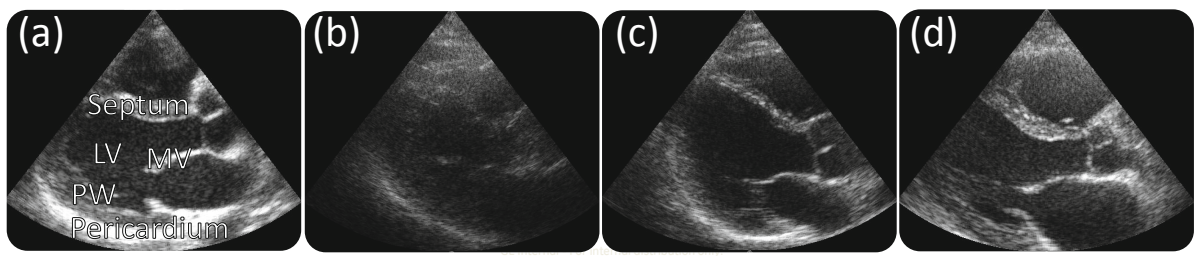

Fig. 1. Examples of good (a) and bad (b,c,d) quality Parasternal Long Axis (PLAX) images. Key: LV - left ventricle, MV - mitral valve, PW - posterior wall.

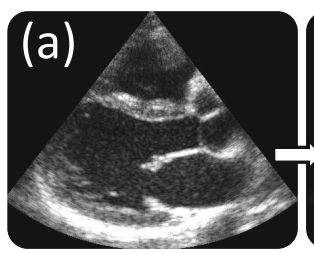

Original image

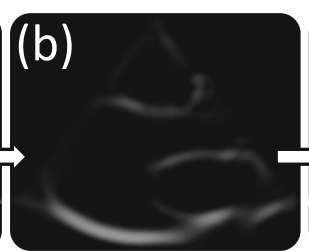

Contrast-enhanced image

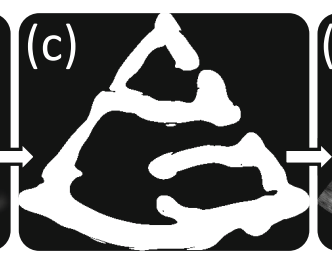

Segmented image

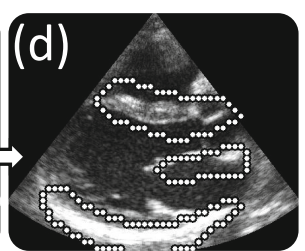

GHT

Fig. 2. Flow chart illustrating the proposed methodology to compute the quality metric

compensation. The non-parallel septum and pericardium in Fig. 1(c) tells us that the imaging plane did not pass through the center of left ventricle. In Fig. 11(d), the pericardium is missing, thereby, complicating the measurement of the thickness of posterior wall and diagnosis of pericardial effusion.

The intuition behind our algorithm is to check for the presence of three prominent tube-like structures corresponding to septum, mitral valve and pericardium. As visualized in Fig. 2, the proposed approach comprises of the following three steps. 1) A pre-processing step that enhances the contrast of thick tube-like structures. 2) A global thresholding step, that outputs a binary image which includes the three structures of interest: septum, mitral valve and pericardium. 3) A search algorithm based on Generalized Hough Transform (GHT) 3] 4, that best matches a pre-defined atlas with the binary image. The GHT outputs an accumulator image, whose maximum value is output as the PLAX Quality Metric (PQM).

\section{Methodology}

In the following, we present an algorithm that searches for septum, mitral valve, and the pericardium, and outputs PQM, which is indicative of how prominently these structures appear in the image.

Contrast Enhancement and Segmentation: As noted by Boukerroui et al. [5], segmentation algorithms based only on global information such as thresholding techniques, intensity dependent clustering and edge detection schemes 
give poor results on ultrasound images. The reason being, the echo amplitude varies according to the orientation of the reflected structure and the image settings such as gain, dynamic range, or time-gain compensation. This is commonly referred to as the intensity inhomogeneity problem.

To mitigate intensity inhomogeneity, we filter the input echo image using Frangi vesselness filter [6] to obtain an intermediate image as shown in Fig. 2(b). The filtered image has less intensity inhomogeneity, and we have observed that global thresholding schemes are more successful on this intermediate representation.

Frangi's vessel enhancement filter is based on the eigen analysis of the Hessian matrix of image intensity at each pixel location of the image. The eigen analysis of the Hessian directly gives the direction of smallest curvature (along the tubular structure). The mutual magnitude of eigenvalues is indicative of the shape of the underlying object. Frangi et al.noted that if a pixel were to lie on a tubular structure, then, one of the eigenvalues has much higher magnitude than the other. If the pixel was from a background region, then both eigenvalues will have low magnitudes.

Let $\lambda_{1}$ and $\lambda_{2}$ denote the two eigenvalues the Hessian $\mathcal{H}_{o, s}$, computed at scale $s$ at pixel $x_{o}$. Frangi et al. propose the following equation to obtain a vesselness image $(\mathcal{V})$.

$$
\mathcal{V}_{o}= \begin{cases}0 & \text { if } \lambda_{2}>0 \\ \exp \left(-\frac{\mathcal{R}_{B}^{2}}{2 \beta^{2}}\right)\left(1-\exp \left(-\frac{\mathcal{S}^{2}}{2 c^{2}}\right)\right) & \text { otherwise }\end{cases}
$$

Here $\mathcal{R}_{B}=\lambda_{1} / \lambda_{2}, \mathcal{S}=\sqrt{\lambda_{1}^{2}+\lambda_{2}^{2}}, \beta$ and $c$ are constants which we set to 1 and 0.5 , respectively. We experimentally observed that by thresholding $\mathcal{V}$ at 0.02 , one obtains a binary image as illustrated in Fig. 2(c).

Commonly, depth parameter of the echocardiogram is set such that the pericardium is at the bottom of the image for PLAX images. We resized all PLAX images to $50 \times 50$ pixels, and we noted that the thickness of pericardium, mitral value, septum does not vary much. Therefore, we chose to detect vessels at one scale $(s=6$ pixels). Although vascular enhancement at multi-scales might produce better images, we chose a single scale to reduce the processing time.

Note that the underlying theory behind this preprocessing step is not specific to vessels. A popular extension of Frangi's technique by Antiga [7 is capable of enhancing contrast of blob-like and plate-like structures.

Generalized Hough Transform (GHT): It is essentially a method to detect the presence of an arbitrary object (described with its model/atlas) in a binary image [3 4. In our case, the atlas is illustrated in Fig. 3(b), and the image to be searched for is the binary image obtained after thresholding the Frangi vesselness image (Fig. 2(c)).

The GHT uses a lookup table that encodes the relationship between the atlas and the Hough parameters. This lookup table is called the R-Table, and the Hough parameters, $r$, and $\alpha$ are computed during the training phase. See Algorithm 1 for details. The parameter $r$ encodes the distance between a pixel and 


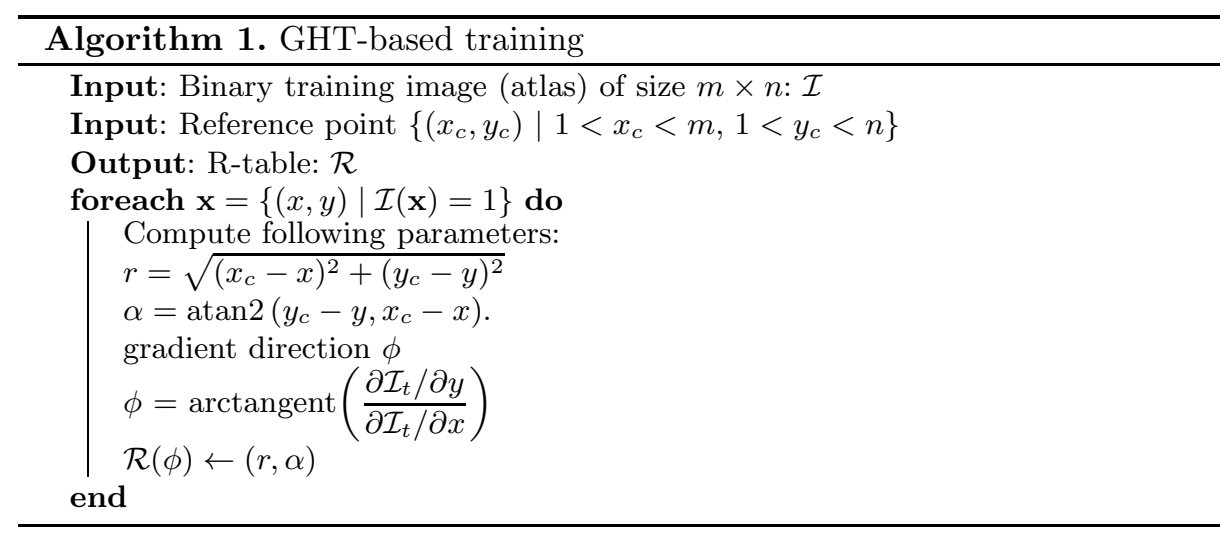

the reference point (which is chosen arbitrarily within the image). The parameter $\alpha$ encodes the angle, in radians, between the positive $\mathrm{x}$-axis and the vector spanning a pixel and the reference point. During the matching phase, as shown in Algorithm 2, one attempts to find the most probable location of the atlas on a test image. The accumulator $\mathcal{A}$ is a $2 \mathrm{D}$ array that holds the votes received for the most probable location of the reference point. The pixel with the maximum intensity in $\mathcal{A}$ is the most probable location of the reference point. The maximum value of $\mathcal{A}$ is output as PLAX Quality Metric (PQM).

Although correlation based techniques [8] are a viable alternative to GHTbased matching, the choice of GHT was based on the interest in speeding up the search procedure. If the atlas and the segmented image have $n$ pixels, then the correlation based procedure, in spatial domain, has the complexity $\mathrm{O}\left(n^{2}\right)$. In contrast, the GHT-based procedure has the complexity $\mathrm{O}\left(m^{2}\right)(m<<n)$, where $m$ is the number of white pixels in atlas and the segmented image.

PLAX Atlas: As mentioned above, GHT matching procedure (Algorithm 2) uses an atlas that defines the structures of interest in PLAX images. The atlas was generated via the following steps.

1. Manual segmentation of the regions of interest in PLAX images. An example is shown in Fig. 31(a).

2. Average representation of the structures of interest was obtained using Shape-Based Averaging (SBA) algorithm [9].

Several independent binary images result from the manual segmentation process, which must be somehow combined into a single final segmentation. Majority voting is the generally used rule to fuse the segmentations, but better methods, such as SBA, have been proposed. SBA consists of averaging Euclidean distance maps computed for all structures for each candidate manual segmentation. The method was shown to keep structure regularity and contiguity better than majority voting. From 89 manually segmented frames, we obtain a PLAX atlas as illustrated in Fig. 3 (b). 

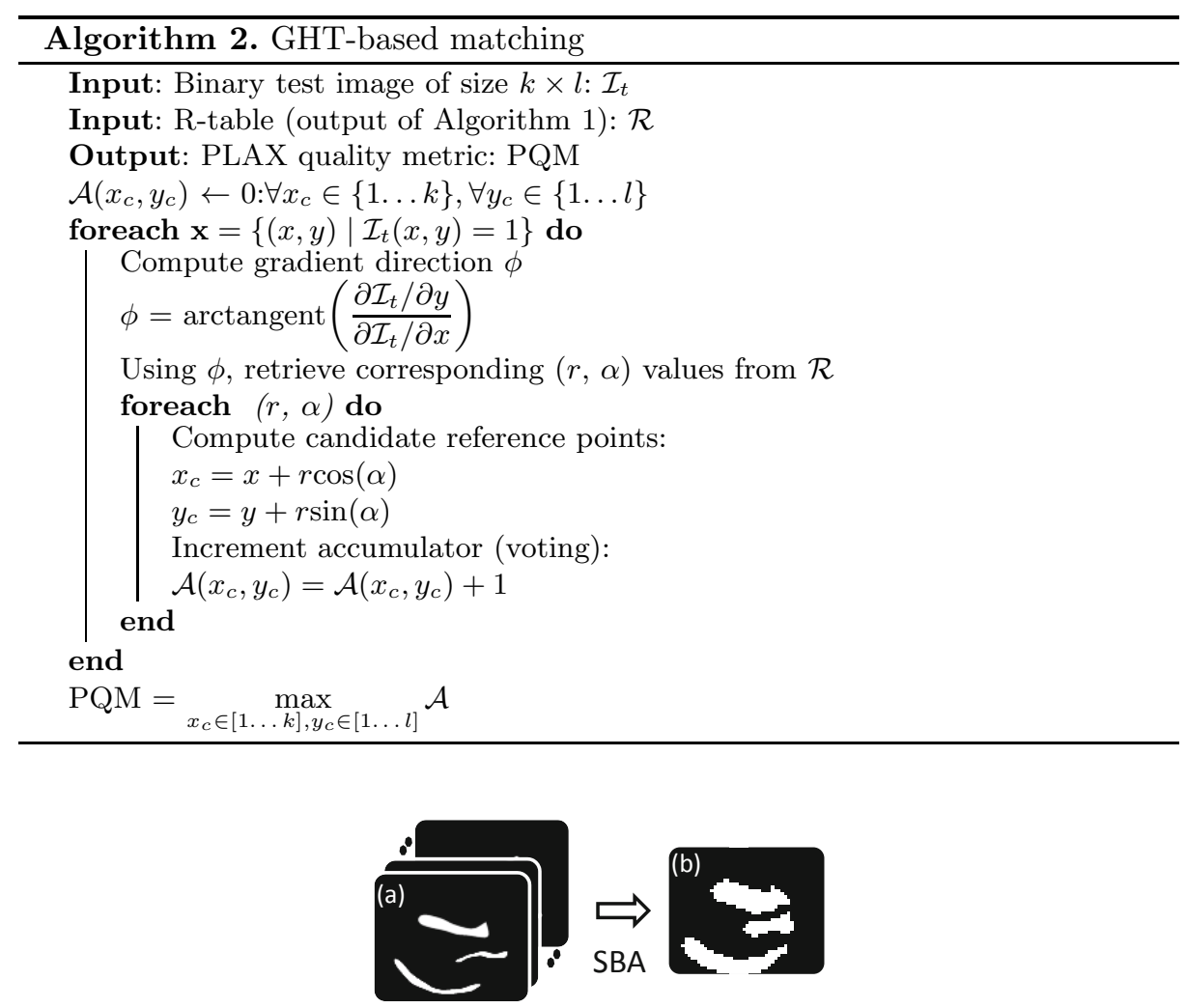

Fig. 3. A total of 89 manually segmented images (a) belonging to distinct patients were used to obtain the PLAX atlas (b)

\section{Results}

A total of 35 subjects with varied clinical background, normal chamber dimensions and normal systolic function underwent routine echocardiography (commercially available Vivid 7, GE) with electrocardiogram gating. The patient data used in our validation included normal and hypertrophic patients. The data was acquired by both echo-cardiologist and an echo-technician at a clinical site. PLAX images with 3 cardiac cycles were analyzed by an expert sonographer for grading image quality.

Comparison with Manual Ratings: The purpose of this experiment was to verify whether PQM correlates with an expert echocardiographist's ratings. The expert manually rated the quality of septum, mitral valve and pericardium on 133 PLAX echocardiograms belonging to 35 patients. Each of these components was given a rating between 0 and 3 , with 0 signifying poor visibility and 3 signifying good visibility. The final manual score for an image was obtained as the average 

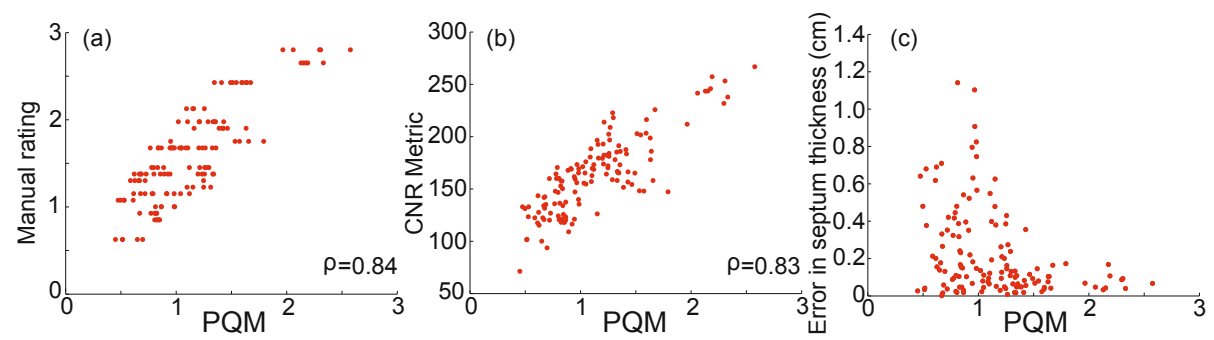

Fig. 4. Comparison with Contrast-to-Noise ratio. Scatter plot between Contrast-toNoise (CNR) ratio and PQM. Scatter plot between error in septum thickness measurement and PQM.

of the individual scores. Thus, the manual score for an image varies between 0 and 3. To facilitate comparison with automatic ratings, we applied min-max normalization to the automatic ratings such that the value of PQM is between 0 and 3. Fig. 4(a) shows a plot between manual rating and PQM. As observed, PQM seems to correlate well (Pearson's correlation coefficient, $\rho=0.84$ ) with an echocardiographist's manual ratings.

Comparison with Contrast-to-Noise Ratio: Contrast-to-noise ratio (CNR) has been traditionally used in medical imaging community to quantify the quality of acquired images. This metric removes the subjectivity factor from the manual ratings. CNR measures the ability to distinguish between an object of interest and its surroundings. The CNR can be defined as: CNR $=\frac{\left|\mu_{o}-\mu_{s}\right|}{\sigma}$. Here, $\mu_{o}$ and $\mu_{s}$ are the mean pixel intensities in the object and surrounding regions, respectively. The quantity $\sigma$ is the standard deviation of the intrinsic noise of the imaging system.

Using manual segmentations of septum, mitral valve and pericardium (See Fig. 3(a) for an illustration), we obtained masks over the regions of interest, and thus, we could compute $\mu_{o}$ for each image. The immediate surrounding areas of the mask were used to compute $\mu_{s}$. The noise parameter, $\sigma$, can be effectively ignored for our experiments because all the images were acquired using the same ultrasound machine.

On 133 PLAX echocardiograms, the CNR metric and the PQM were computed. The results are shown as a scatter plot between the two quantities in Fig. 4(b). We observed a Pearson's correlation coefficient, $\rho$, of 0.83.

Comparison with Error in Septum Thickness Measurement: Subramanian et al. 10 proposed a snakes-based approach that automatically measures septal wall thickness according the existing clinical guidelines [11]. In an attempt to verify if PQM can be used to predict accuracy of septal wall thickness measurement algorithm, we visualized the error in thickness measure and PQM as a scatter plot in Fig. 4(c). We observed that on images with low PQM, the probability of error in septum thickness measurement will be high and vice versa. 


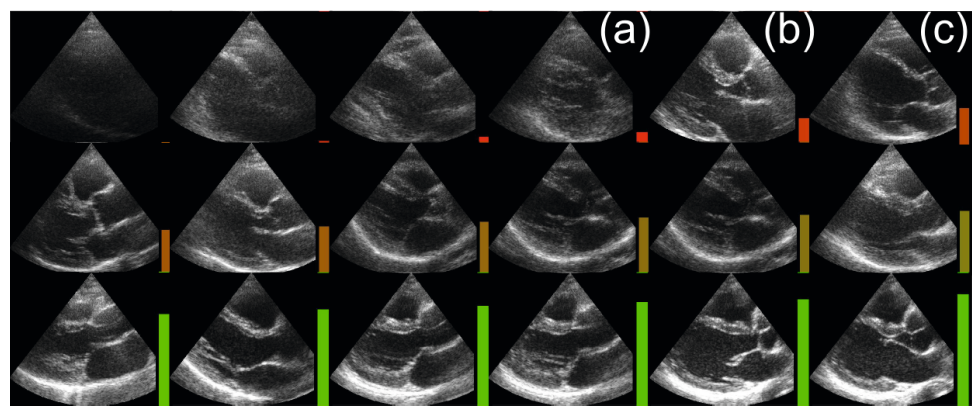

Fig. 5. PQM, illustrated as vertical bars, computed on few test images. The height of the bar is proportional to PQM. Note that PQM is low for (a), (b), and (c), which represent the bad quality PLAX images illustrated in Fig. 1. As the anatomical components of the heart become progressively more visible, PQM increases.

Thus, one could deploy automatic segmentation algorithms only when PQM is greater than a preset threshold, thereby, providing an upper bound on the segmentation error.

Visual Assessment of PQM: Fig. 5 visualizes PQM as vertical bars. The height of the bar is proportional to the PQM computed for an image. Typical poor quality PLAX echocardiograms are shown in sub-figures (a), (b) and (c). The contrast between the blood pool and the structures of interest is low in (a). Pericardium is not visualized in (b). Sub-figure (c) illustrates the case where septum and pericardium are not parallel to each other, and this signifies an incorrect scan plane. Note that PQM is low for all three cases. For images with better quality, one sees an increase in PQM.

\section{Conclusions and Discussion}

In this paper, we proposed a solution to the previously unstudied problem of computing a quality metric for Parasternal Long AXis (PLAX) view echocardiograms. Our algorithm checks for the presence of expected anatomical structures in a PLAX image (Septum, mitral valve and pericardium) using Generalized Hough Transform (GHT). Based on the evidence GHT accumulates during the search, we output PQM that seems to correlate well with an expert's rating and CNR metric. We also observe that for images with high PQM, error in septum thickness measurement algorithm decreases.

Because the expected structures in a PLAX image are relatively large, we observed that we could subsample the image to $50 \times 50$ pixels, and still see the structures of interest. At this resolution, we could obtain a processing speed of 35 frames a second in a Intel i7 $2.67 \mathrm{GHz}$ processor. The implementation was done in $\mathrm{C}++$ using the Insight Segmentation and Registration Toolkit [12]. The results illustrated in this paper have been performed by resizing images to $50 \times 50$ pixels. 
The proposed quality metric is the first step in guiding the user into acquiring the right scan plane. The user will receive real-time feedback to the acquired image, thereby, motivating the user to acquire a better quality image. Although, the proposed method is specific for PLAX images, we perceive easy extension to other anatomies using an appropriate atlas and a suitable contrast enhancement technique.

\section{References}

1. Alonso-Fernandez, F., Fierrez-Aguilar, J., Ortega-Garcia, J.: A review of schemes for fingerprint image quality computation. In: Cost-275 Workshop, pp. 3-6 (2005) 1

2. Goodsitt, M., Carson, P., Witt, S., Hykes, D., Kofler, J.: Real-time B-mode ultrasound quality control test procedures. Medical Physics 25, 1385-1406 (1998) 1

3. Duda, R., Hart, P.: Use of the Hough transformation to detect lines and curves in pictures. Communication ACM 15, 11-15 (1972) 2, 3

4. Ballard, D.: Generalizing the Hough transform to detect arbitrary shapes. Pattern Recognition 13, 111-122 (1981) 2, 3

5. Boukerroui, D., Baskurt, A., Noble, J., Basset, O.: Segmentation of ultrasound images. Pattern Recognition Letters 24, 779-790 (2003) 3

6. Frangi, A.F., Niessen, W.J., Vincken, K.L., Viergever, M.A.: Multiscale Vessel Enhancement Filtering. In: Wells, W.M., Colchester, A., Delp, S. (eds.) MICCAI 1998. LNCS, vol. 1496, pp. 130-137. Springer, Heidelberg (1998) 3

7. Antiga, L.: Generalizing vesselness with respect to dimensionality and shape. The Insight Journal (2007) 3

8. Gonzalez, R., Woods, R.: Digital image processing, ch. 9 (1993) 4

9. Rohlfing, T., Maurer, C.: Shape-based averaging. IEEE Transactions on Image Processing 16, 153-161 (2007) 4

10. Subramanian, N., Padfield, D., Thiruvenkadam, S., Narasimhamurthy, A., Frigstad, S.: Automated Interventricular Septum Thickness Measurement from BMode Echocardiograms. In: Jiang, T., Navab, N., Pluim, J.P.W., Viergever, M.A. (eds.) MICCAI 2010, Part I. LNCS, vol. 6361, pp. 510-517. Springer, Heidelberg (2010) 7

11. Lang, R., Bierig, M., Devereux, R., Flachskampf, F., Foster, E., Pellikka, P., Picard, M., Roman, M., Seward, J., Shanewise, J., Solomon, S., Spencer, K., Sutton, M., Stewart, W.: Recommendations for chamber quantification. European Journal of Echocardiography 7, 79-108 (2006) 7

12. Ibáñez, L., Schroeder, W., Ng, L., Cates, J.: The ITK Software Guide. Kitware (2003) 8 